\title{
Pyomyositis in Spinal Cord Injury Patients
}

\author{
M. Midha, MD, ${ }^{1}$ R. Hussey, $M D,{ }^{2}$ D. A. Sica, $M^{3}$ \\ ${ }^{1}$ Staff Physician, ${ }^{2}$ Chief, Spinal Cord Injury Service, McGuire VA Hospital, \\ Richmond, Virginia, USA, ${ }^{3}$ Associate Professor of Medicine Chief Renal Phar- \\ macology Section, Division of Nephrology, Medical College of Virginia, Richmond, \\ Virginia, USA.
}

\section{Summary}

Bacterial infections remain a major cause of morbidity and mortality among patients with longstanding spinal cord injury. Infection may occur in a number of different foci, often taxing the skill of the most accomplished diagnostician. Herein are described three patients with fever of unknown origin wherein extensive diagnostic workups ultimately led to the diagnosis of primary pyomyositis. These patients demonstrate the importance of repeated careful physical examination in determining the site of infection in patients with established sensorimotor deficits. Although primary pyomyositis is not a common occurrence in those with spinal cord injuries, it should be included as a potential cause of fever in this patient population.

Key words: Primary pyomyositis; Sensory loss; Spinal cord injury patients.

Pyomyositis is a pyogenic infection of skeletal muscle the commonest of which is Staphylococcus aureus (Chiedozi, 1979). Although originally described almost one century ago (Scriba, 1885), its development and treatment remain obscure, with few physicians having experience with either its diagnosis or management. Reports of pyomyositis in the iliopsoas muscle, secondary to the extension of inflammatory processes from contiguous structures are common. Primary pyomyositis, on the other hand, is a relatively rare condition. It most frequently occurs in tropical and subtropical areas where it may account for up to $4 \%$ of surgical hospitalisations (Horn, 1968). For unknown reasons it is rare in temperate climates, and its clinical diagnosis is often delayed because of a lack of familiarity with the condition.

Three case reports of primary pyomyositis in spinal cord injured (SCI) patients are herein presented. Signs and symptoms of infection in this patient population are frequently masked secondary to sensorimotor deficits and the liberal use of antibiotic therapies. Such alterations in the presenting manifestations of 
infectious illnesses oftentimes contributes to their indolent nature and are illustrated by the disease course in our 3 patients.

\section{Case report 1}

This 34-year-old T4 complete paraplegic patient secondary to a gunshot injury in February 1983 was admitted to our service on 10 October 1983 for management of spasticity and depression. On 12 December the patient developed chills and a fever of $103^{\circ} \mathrm{F}$ associated with an increased WBC of 18500 and a leftward shift. Repeated clinical examinations, blood cultures, and urine cultures were negative. Chest $\mathrm{X}$-ray, abdominal ultrasound, intravenous pyelogram, gallium scan and bone scan were all negative. Although no infecting focus could be demonstrated, the patient was placed on piperacillin and amikacin on clinical grounds. On 22 December 1983, a swelling in the left thigh was noticed that had not been present on earlier examinations. No preceding trauma in this area was reported nor had any intramuscular injections been given in the region. Ultrasound of the thigh revealed a cylindrical area of low echo density under the fascial plane extending from the superior portion of the medial thigh to near the knee joint. Under ultrasonic guidance $360 \mathrm{cc}$ of purulent foul smelling material was aspirated. The gram stain was positive for gram negative rods though no organism was grown on either aerobic or anaerobic culture. CT scan of the abdomen was performed to rule out any retroperitoneal connection and it was negative. On that same day the patient underwent incision and drainage of the left medial thigh which showed massive necrosis of the adductor muscle. The patient became afebrile 1 hour post-operatively and remained so. After local wound care the wound was closed secondarily on 12 February 1984, and healed per primum.

\section{Case report 2}

This 41-year-old C7 quadriplegic whose injury resulted from an automobile accident in April 1981, was admitted on 27 April 1983, with a main complaint of fever of 2 or 3 days' duration, bilateral trochanteric ulcers, a grade three sacral ulcer, and multiple lesions over the spinous processes and both lateral malleoli. The patient was severely anaemic and cachectic. A fever 'work-up' discovered a Pseudomonas urinary tract infection. This organism was sensitive to amikacin to which the patient responded clinically. He subsequently underwent closure of the right trochanteric ulcer with a tensor fascia femoris flap on 22 July 1983, and closure of the sacral sore with a rotational flap on 28 October 1983. He developed three episodes of urinary tract infection between October 1983 and January 1984. In each instance the infection resolved with the institution of appropriate antibiotics. On 28 January 1984, the patient had a fever of $102^{\circ} \mathrm{F}$ associated with swelling of the right thigh. Aspiration of the right thigh swelling was carried out, and purulent material was obtained which on gram stain showed gram positive cocci and polymorphonuclear leukocytes. Culture of the pus grew Staphylococcus aureus resistant to penicillin. Incision and drainage was performed on the medial right thigh 4 inches below the groin proximally and 6 inches above the knee distally on 29 January 1984, whilst the patient was receiving nafcillin. At the time of surgery a large abscess cavity was found with surrounding adductor muscle necrosis. The thigh wound was left open over a prolonged time period to facilitate local care, and closure was eventually accomplished by secondary intention in May 1984. The patient has not had any fever or recurrence of the abscess.

\section{Case report 3}

This 25-year-old T6 complete paraplegic patient, due to a motor vehicle accident on 10 October 1985, was admitted on 8 October 1986 with a main complaint of fever $\left(103^{\circ} \mathrm{F}\right)$, chills and a swelling of the right medial thigh for the preceding 4 days. 


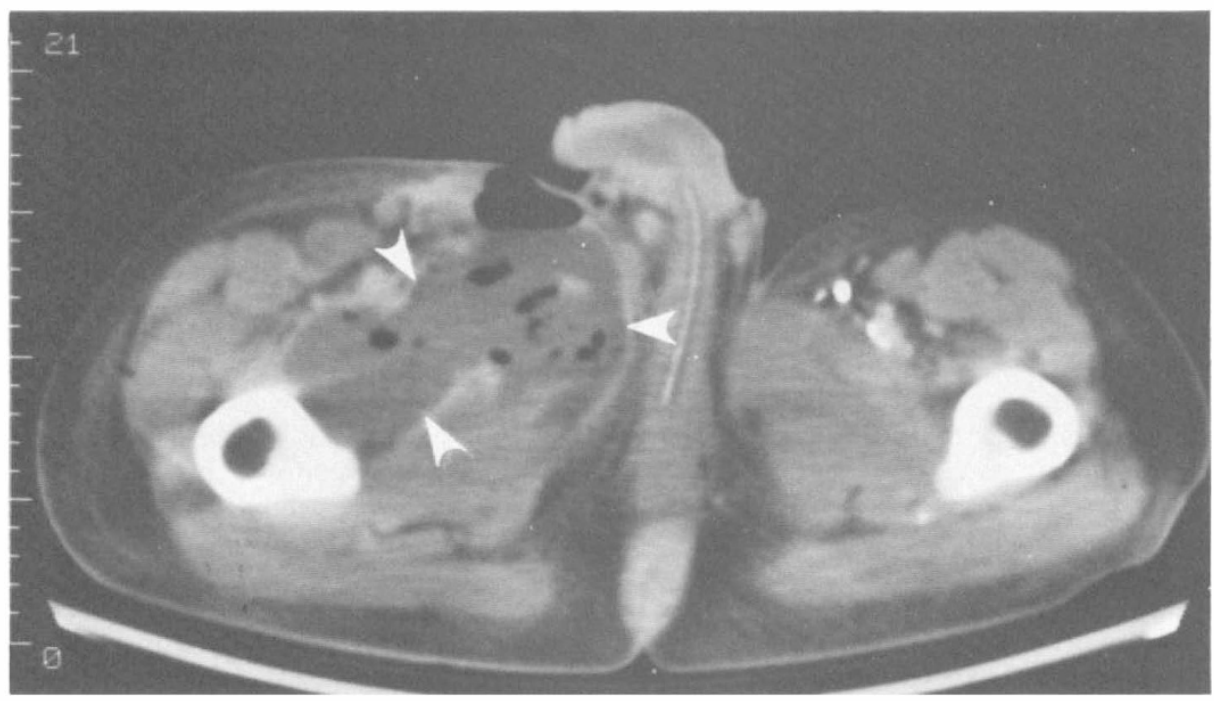

Figure 1 Large soft tissue abscess involving the lower pelvic area (white area) as determined by CT scan in patient 3 .

On admission, the physical examination was remarkable for a $\mathrm{T} 6$ complete paraplegic patient in that there was a swelling in the right medial thigh region which extended from the groin to the knee. The admission WBC count was 27100 and a urinalysis was $4^{+}$for bacteria with $5-10 \mathrm{WBC} / \mathrm{hpf}$ and $10-12 \mathrm{RBC} / \mathrm{hpf}$ (the patient was on external urinary drainage). A chest X-ray, KUB and right femur film were all negative. Blood cultures on admission were negative whilst a urine culture grew $E$. Coli. The patient was initially started on nafcillin based on the presumption of right thigh cellulitis. On 9 October 1986 a CT of the pelvis and right thigh revealed a large abscess involving the superficial anterior soft tissue of the right lower pelvis with extension deep into the posterior medial aspect of the right thigh to just above the knee joint (Fig. 1). There was no evidence of osteomyelitis, and there were no peritoneal or retroperitoneal fluid collections. On 10 October 1986 a needle aspiration of the right thigh revealed pus which grew E. Coli. At this point cephapirin was started but, despite this, a hectic fever course continued. On 15 October 1986 the right thigh abscess was incised and drained. At the time of surgery extensive adductor muscle involvement was noted. The patient subsequently became afebrile within 48 hours. Subsequently the wound is being managed with local wound care (Fig. 2) and will be closed by secondary intention.

\section{Discussion}

Fever, infection or both are frequent occurrences during hospitalisation of a spinal cord injured patient (Sugarman, 1983). Urinary tract and soft-tissue infections tend to predominate with multiple organisms often being involved (Sugarman, 1982; Vaziri, 1982). The occurrence of soft-tissue infections commonly relates to the development of a decubitus ulcer or to spread from adjoining infected bony structures. The development of a primary pyogenic skeletal muscle infection, pyomyositis, is distinctly uncommon in this patient population and its discovery requires considerable diagnostic acumen.

The pathogenesis of pyomyositis has not been completely elucidated. A unifying etiologic factor does not exist although trauma, vibrio infection, parasitic 


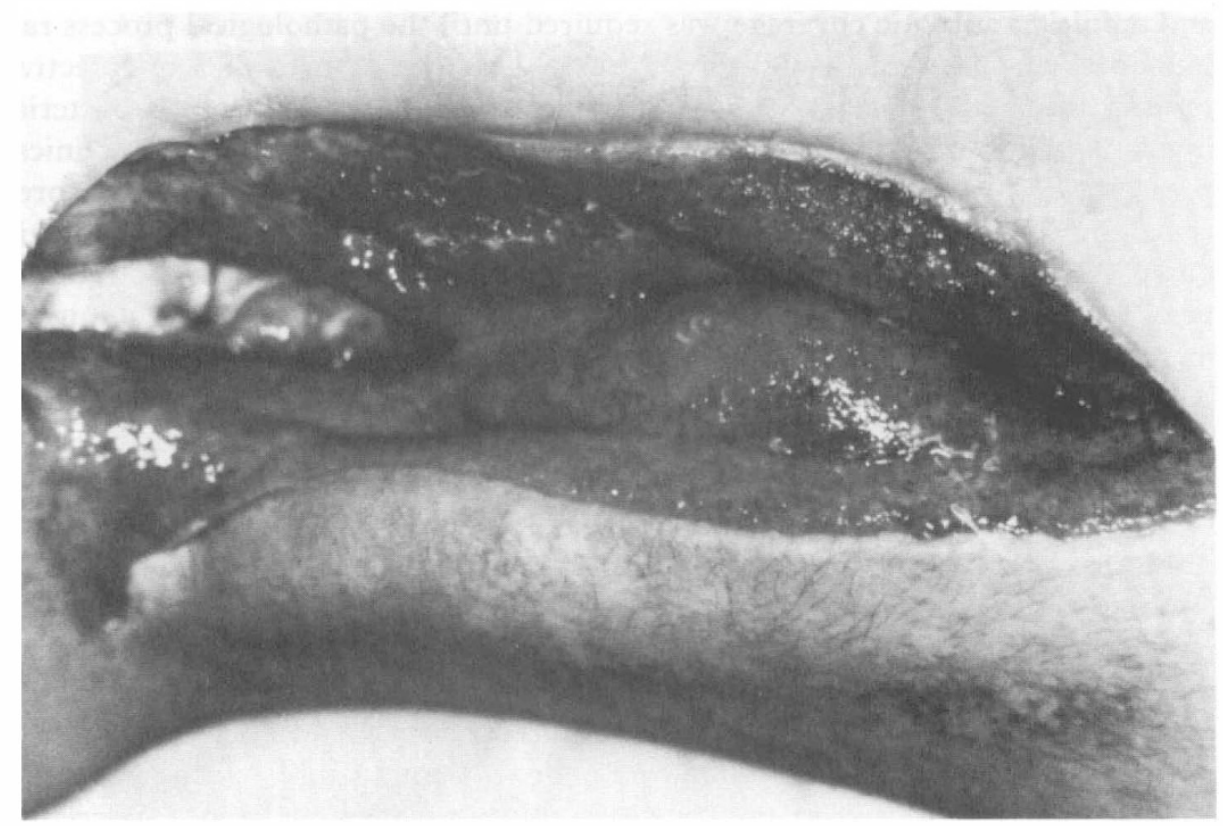

Figure 2 Appearance of thigh region in patient 3 subsequent to surgical debridement of involved area.

infection, and hemoglobinopathies have all been implicated as possible predisposing conditions (Altrocchi, 1971). However, the present report concerns itself with bacterial myositis occurring spontaneously without an established antecedent cause.

Bacterial myositis usually presents subacutely with moderate muscle pain. It is often preceded by several days to weeks of fever although the disease can occasionally begin as an acute toxic illness with high fever and chills (Chaitow, 1980; Altrocchi, 1971) as was the case in all three patients. Local lymph nodes are not usually enlarged and blood cultures are seldom positive. Clinically, a solitary muscle abscess is more common than multiple abscesses. In its early stages the differential diagnosis of this illness includes osteomyelitis, thrombophlebitis, muscle rupture or muscle hematoma (Chiedozi, 1979).

Pyomyositis has a predilection for large muscle masses of the body such as those of the upper thigh which are ordinarily resistant to bacterial invasion. The abscesses occur deep within a large muscle and remain well localised due to overlying muscle fascia, thus sparing the subcutaneous and cutaneous tissue until the later stages of the illness. Consequently, pain is rarely a significant symptom, and erythema and heat may be minimal or absent. It is only after several days to weeks that erythema and fluctuation appear. The diagnosis of pyomyositis becomes even more difficult in the SCI population since neuromuscular and sensory deficits limit the early expression of signs and symptoms of this particular disease. Of our three patients, only case 3 had a localising sign (right thigh swelling). Despite this, the pattern of diagnosis and treatment was quite similar in all three patients in that a careful 'fever workup' was carried out 
and empiric antibiotic coverage was required until the pathological process ran its course and presented clinically. In case 1 there was no apparent infective process that could have served as the primary cause of the observed bacterial myositis which permitted the diagnosis of primary pyomyositis. The clinical presentation in case 2 was more complex since the bacterial myositis was preceded by soft-tissue infection in the trochanteric area several months previously. Although it is unlikely, the trochanteric ulcer which occurred 6 months prior to the myositis may have served as an initiating nidus for eventual muscle infection. We think this to be highly unlikely, based on the time span between both events and feel that a diagnosis of primary pyomyositis is justified. In case 3 once again there was no primary infective process to which the myositis could be attributed.

Irrespective of the title affixed to this form of bacterial myositis, surgical drainage of all the abscesses is the sine qua non of management. As illustrated in all three of our patients fever disappeared within 48 hours of incision and drainage, and clinical improvement rapidly followed. The gratifying clinical response in these patients justifies the considerable effort expended in the course of evaluating the fever in the spinal cord injured patient, and once again points out that fever in the spinal cord injured patient is not a simple event. Although antibiotics are often utilised empirically in this population, in time, a source for the fever will be discovered although it can be in an occult location.

\section{References}

Altrocchi PH 1971 Spontaneous bacterial myositis. Fournal of the American Medical Association 217:819-820.

Chaitow J, Martin HCO, Knight P, et al. 1980 Pyomyositis tropicans: a diagnostic dilemma. Medical fournal of Australia 2:512-513.

CHIEdozi LC 1979 Pyomyositis: review of 205 cases in 112 patients. American fournal of Surgery 137:255-259.

HoRn CV, MASTER S 1968 Pyomyositis tropicans in Uganda. East African Medical fournal 45:463-471.

SCRIBA J 1985 Beitrag zur Aetiologie der Myositis acuta. Deutsch Zeitschrift fur Chirurgie 22:497502.

Sugarman B, Borwn D, Musher D 1982 Fever and infection in spinal cord injury patients. fournal of the American Medical Association 248:66-70.

VAZIRI ND, Cesario T, MоOToo K, et al. 1982 Bacterial infections in patients with chronic renal failure: occurrence with spinal cord injury. Archives of Internal Medicine 142:1272-1276. 